\title{
Towards generalised diffraction integration software: neutron diffraction analysis in DIALS
}

\author{
D. McDonagh ${ }^{1}$, D. Waterman ${ }^{1,2}$ \\ ${ }^{1}$ STFC Rutherford Appleton Laboratory, Didcot, OX11 OFA, England, \\ ${ }^{2}$ CCP4, Research Complex at Harwell, Rutherford Appleton Laboratory, Didcot OX11 OFA, England
}

david.mcdonagh@stfc.ac.uk

The DIALS project [1] provides an open-source, extensible framework to analyse X-ray diffraction data and is now used widely in the X-ray diffraction community. Much of this framework is in principle agnostic to the method used to obtain diffraction patterns. In recent years this has been expanded for stills shot serial crystallography and continuous-rotation electron diffraction experiments [2], for example, highlighting how DIALS can be adapted to cope with challenges from electron sources such as low diffraction angles and lens distortion. Continuing with this push towards generalised diffraction integration software, here we present preliminary results for processing time-of-flight neutron diffraction patterns obtained from the Single Crystal Diffractometer (SXD) at ISIS [3].

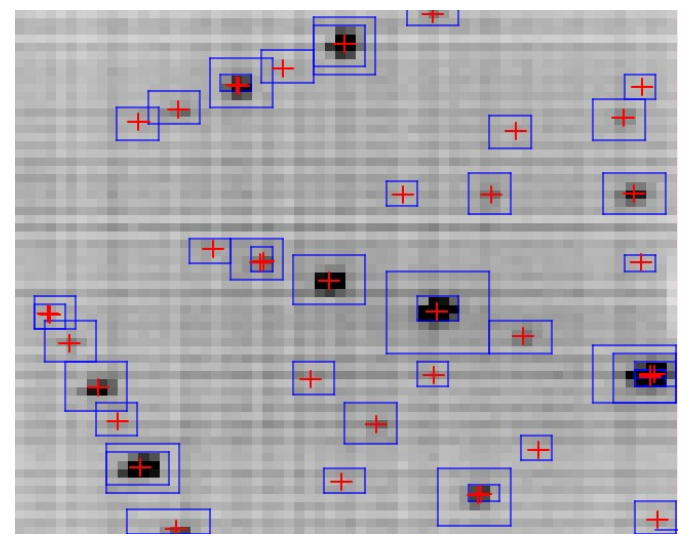

Figure 1. An example of the output of the DIALS image viewer on a single SXD panel after running the DIALS find spots algorithm. Bragg peaks are shown in black, with their calculated centroids shown as red crosses and shoeboxes shown in blue. For clarity the time-of-flight dimension has been summed to give a $2 \mathrm{D}$ plot.

Neutron diffraction provides a complimentary technique to X-ray diffraction, exploiting nuclei interactions to render isotopes and light atoms that are generally absent from X-ray data. A lack of sample radiation damage further allows for the same crystal to be used for both X-ray and neutron diffraction, and such joint neutron/X-ray approaches are increasingly common for macromolecular crystallography [4]. By incorporating neutron diffraction into DIALS, we provide a common interface for users working with different diffraction sources to draw on the expanding toolbox of DIALS algorithms. This is further emphasised by providing the ability to convert DIALS output to formats readable by other related software, in this case Mantid [5], greatly increasing the transferability of different analysis tools. Here we show how DIALS can be used for the first time for time-of-flight neutron diffraction data, allowing Bragg peaks at a variety of wavelengths to be identified and visualised in the DIALS image (Fig. 1) and reciprocal lattice viewers, indexed, and refined to identify the sample space group. We also outline how DIALS refinement and integration is being adapted to cope with polychromatic data from pulsed neutron sources.

[1] Winter G., Waterman D. G., Parkhurst J. M., Brewster A. S., Gildea R. J., Gerstel M., Fuentes-Montero L., Vollmar M, Michels-Clark T., Young I. D., Sauter N. K., Evans G. (2017). Acta Crystallogr. D. 74, 85-97

[2] Clabbers T. B., Gruene T., Parkhurst J. M., Abrahams J. P., \& Waterman D. G. (2018). Acta Crystallogr. D. 74, 506-518

[3] Keen D. A, Gutmann M. J., \& Wilson C. C. (2006). J. Appl. Cryst. 39, 714-722

[4] Blakeley M. P. \& Podjarny A. D. (2018). Emerg. Top. Life Sci. 2, 39-55

[5] Arnold O., et al. (2014). Nucl. Instrum. Methods Phys. Res. 764, 156-166

Keywords: DIALS; software; neutron diffraction; time-of-flight; integration; refinement; joint refinement 